\title{
SOME POINTS IN THE THEORY OF TRIGONOMETRIC AND POWER SERIES*
}

\author{
BY \\ ANTONI ZYGMUND
}

\section{ON THE CHARACTER OF OSCILLATION OF THE PARTIAL SUMS OF FOURIER SERIES}

1. The fundamental theorem. Completing a well known result of Kolmogoroff [12], Marcinkiewicz [15] has recently constructed a function integrable $L$, whose Fourier series possesses partial sums oscillating finitely almost everywhere. It is, therefore, natural to ask what may be said about the relative position of the interval of oscillation of $s_{n}(x)$ and the value $f(x)$, beyond the well known fact that the said interval contains $f(x)$. The result proved in this note is a first attempt in this direction.

ThEOREM. If the partial sums $s_{n}(x)$ of the Fourier series of a function $f(x)$ integrable $L$,

$$
f(x) \sim \frac{1}{2} a_{0}+\sum_{n=1}^{\infty}\left(a_{n} \cos n x+b_{n} \sin n x\right),
$$

satisfy an inequality

$$
s_{n}(x) \geqq-\phi(x) \quad(0 \leqq x \leqq 2 \pi ; n=0,1,2, \cdots),
$$

with $\phi(x) \geqq 0$ integrable $L$, then, for almost every $x$,

$$
f(x)=\frac{1}{2}\left[\limsup _{n \rightarrow \infty} s_{n}(\dot{x})+\liminf _{n \rightarrow \infty} s_{n}(x)\right] .
$$

2. Statement of lemmas. The proof of our theorem is based on three lemmas which will be stated in this section and the proof of which will be given in the next section. Let $\sigma_{n}(x), \tilde{\sigma}_{n}(x), \tilde{s}_{n}(x)$ denote, respectively, the first arithmetic means of the series (1), of the conjugate series

$$
\sum_{n=1}^{\infty}\left(a_{n} \sin n x-b_{n} \cos n x\right),
$$

and the partial sums of the latter series. We have $[20,24,18,30]$

* Presented to the Society, March 31, 1934; received by the editors November 5, 1933. The six notes constituting this paper are independent of one another, although they treat related topics. The numbers in square brackets refer to the Bibliography at the end of the paper. 


$$
\begin{gathered}
s_{n}(x)-\sigma_{n}(x)=\tilde{s}_{n}^{\prime}(x) /(n+1), \\
\frac{\tilde{s}_{n}^{\prime}(x)}{n+1}=\frac{2}{\pi} \int_{-\pi}^{\pi} s_{n}(u) \cos (n+1)(u-x) K_{n}(u-x) d u,
\end{gathered}
$$

where

$$
K_{n}(u)=\frac{1}{2(n+1)}\left(\frac{\sin \frac{n+1}{2} u}{\sin \frac{u}{2}}\right)^{2}
$$

is the well known Fejér's kernel.

LEMMA 1. Let $H$ be a measurable set contained in $(-\pi, \pi)$ and having $x=0$ as a point of zero density. Then the function

$$
L_{n}(t)=\int_{H} K_{n}(u) K_{n}(u-t) d u
$$

satisfies the relations*

$$
\begin{aligned}
& L_{n}(t) \leqq \frac{C n}{1+n^{2} t^{2}}, \\
& L_{n}(t)=o(n)
\end{aligned}
$$

(uniformly in $t$ ).

LEMMA 2. Under the hypothesis of the theorem we have

$$
\left|\frac{\tilde{s}_{n}^{\prime}(x)}{n+1}\right|=\left|s_{n}(x)-\sigma_{n}(x)\right| \leqq \tau_{n}(x)
$$

where $\tau_{n}(x)$ are the Fejer means of an integrable function $\psi(x) \geqq 0$.

LEMмa 3. If, under the hypothesis of the theorem, we have for every $x$ belonging to a set $E$ of positive measure

$$
s_{n}(x)-\sigma_{n}(x)=\tilde{s}_{n}^{\prime}(x) /(n+1) \geqq-M \quad\left(n \geqq n_{0}, M>0\right),
$$

then, for almost every $x$ in $E$,

$$
\limsup _{n \rightarrow \infty}\left[s_{n}(x)-\sigma_{n}(x)\right] \leqq M .
$$

3. Proof of lemmas. To prove Lemma 1 , let $Q_{n}(u)=n /\left(1+n^{2} u^{2}\right)$. Since the kernel $K_{n}(u)$ is $O(n)$ for $0 \leqq u \leqq 1 / n$, and $O\left(1 /\left(n u^{2}\right)\right)$ for $1 / n \leqq u \leqq 3 \pi / 2$, it is easy to see that $K_{n}(u) \leqq C Q_{n}(u)$ (cf. Fejér [3]). From (5) we deduce that

* In the following we use $C$ as a generic notation for an absolute constant, not necessarily the same in all formulas where it occurs. 


$$
L_{n}(t) \leqq \int_{-\pi}^{\pi} K_{n}(u) K_{n}(u-t) d u .
$$

Since the integral is an even function of $t$, we may restrict ourselves to the values $0 \leqq t \leqq \pi$. Break up the integral into four, extended over the intervals $(-\pi,-\pi / 2),(-\pi / 2,0),(0, t / 2),(t / 2, \pi)$, and denoted respectively by $U_{n}^{(1)}(t), U_{n}^{(2)}(t), U_{n}^{(8)}(t), U_{n}^{(4)}(t)$. Since $Q_{n}(u)$ is decreasing in the interval $0 \leqq u \leqq 3 \pi / 2$, it is readily seen that

$$
\begin{aligned}
& U_{n}^{(1)}(t)=O\left(n^{-1}\right) \int_{-\pi}^{-\pi / 2} K_{n}(u-t) d u=O\left(n^{-1}\right) \leqq C Q_{n}(t), \\
& U_{n}^{(2)}(t) \leqq C Q_{n}(t) \int_{-\pi / 2}^{0} K_{n}(u) d u \leqq C Q_{n}(t), \\
& U_{n}^{(3)}(t) \leqq C Q_{n}(t / 2) \int_{0}^{t / 2} K_{n}(u) d u \leqq C Q_{n}(t), \\
& U_{n}^{(4)}(t) \leqq C Q_{n}(t / 2) \int_{t / 2}^{\pi} K_{n}(u-t) d u \leqq C Q_{n}(t) .
\end{aligned}
$$

Adding these inequalities together we obtain (6).

To obtain (7), it is sufficient to replace in the integral (5) the function $K_{n}(u-t)$ by its upper bound (which is $\left.O(n)\right)$ and to notice that the remaining integral represents the Fejér means, at $x=0$, of the characteristic function of the set $H$, and so, by Lebesgue's well known criterion, tends to 0 with $1 / n$.

To prove Lemma 2 , replace, in the right-hand side of $(4), s_{n}(u)$ by $s_{n}(u)+\phi(u)-\phi(u)$. Then in view of (2), the first term in (8) does not exceed

$$
\begin{gathered}
\frac{2}{\pi} \int_{-\pi}^{\pi}\left[s_{n}(u)+\phi(u)\right] K_{n}(u-x) d u+\frac{2}{\pi} \int_{-\pi}^{\pi} \phi(u) K_{n}(u-x) d u \\
=\frac{1}{\pi} \int_{-\pi}^{\pi} \psi(u) K_{n}(u-x) d u
\end{gathered}
$$

where $\psi(u)=2 f(u)+4 \phi(u)$.

We pass on to the proof of Lemma 3. First, we have, for almost every $x$ in the interval $(-\pi, \pi)$, the relation

$$
\frac{\tilde{s}_{n}^{\prime}(x)}{n+1}=\frac{2}{\pi} \int_{-\pi}^{\pi}\left[s_{n}(u)-\sigma_{n}(u)+M\right] \cos (n+1)(u-x) K_{n}(u-x) d u+o(1) .
$$

For we may replace $s_{n}(u)$ by $s_{n}(u)+M$ under the sign of integral in (4), without changing its value. If we replace there $s_{n}$ by $\sigma_{n}$, we obtain $\tilde{\sigma}_{n}^{\prime} /(n+1)$, which represents the difference, multiplied by $(n+2) /(n+1)$, of the first and second arithmetic means of the series (1). This follows from the formula 


$$
\frac{1}{A_{n}^{(1)}} \sum_{\nu=0}^{n} A_{n \rightarrow \nu}^{(1)} c_{\nu}-\frac{1}{A_{n}^{(2)}} \sum_{\nu=0}^{n} A_{n-\nu}^{(2)} c_{\nu}=\sum_{\nu=0}^{n} \frac{\nu c_{\nu}(n-\nu+1)}{(n+1)(n+2)}=\frac{\tilde{\sigma}_{n}^{\prime}(x)}{n+2},
$$

where $c_{n}$ is the general term of series (1) and

$$
A_{n}^{(k)}=\left(\begin{array}{c}
n+k \\
k
\end{array}\right) \text {. }
$$

This difference obviously tends to zero for almost every $x$

Let now $0<r<1$ be fixed and let

$$
P_{r}(u)=\frac{1}{2}+r \cos u+r^{2} \cos 2 u+\cdots .
$$

Since

$$
\begin{aligned}
\sum_{\nu=0}^{n}\left[\left(1-\frac{\nu}{n+1}\right)-\left(1-\frac{\nu}{n+1}\right)^{2}\right] c_{\nu} \\
=\frac{\tilde{\sigma}_{n}^{\prime}(x)}{n+1}=\frac{1}{\pi} \int_{-\pi}^{\pi}\left[s_{n}(u)-\sigma_{n}(u)\right] K_{n}(u-x) d u,
\end{aligned}
$$

we have

$$
\frac{1}{\pi} \int_{-\pi}^{\pi}\left[s_{n}(u)-\sigma_{n}(u)\right] K_{n}(u-x) d u \rightarrow 0
$$

for almost every $x$, and

$$
\begin{aligned}
& r \frac{\tilde{s}_{n}^{\prime}(x)}{n+1}-M=\frac{2}{\pi} \int_{-\pi}^{\pi}\left[s_{n}(u)-\sigma_{n}(u)+M\right] \\
& \quad \times\left[-\frac{1}{2}+r \cos (n+1)(u-x)\right] K_{n}(u-x) d u+o(1) \\
&=-\frac{2}{\pi} \int_{-\pi}^{\pi}\left[s_{n}(u)-\sigma_{n}(u)+M\right] P_{r}\{(n+1)(u-x)+\pi\} K_{n}(u-x) d u+o(1) .
\end{aligned}
$$

Break up the last integral into two, extended over $E$ and its complement $B$, and denote the corresponding expressions by $J_{1}$ and $J_{2} . P_{r}(u)$ and $K_{n}(u)$ are non-negative, hence by (9), $J_{1} \leqq 0$ and it remains to show that $J_{2} \rightarrow 0$ almost everywhere in $E$. It is sufficient to show that $J_{2} \rightarrow 0$ at every point $x$ where $E$ has density 1 and where the integral of $\psi$ (see Lemma 2) has a finite derivative. Suppose for simplicity that $x=0$ is such a point and let $M_{r}=\max P_{r}(u)$, $0 \leqq u \leqq 2 \pi$. Then (see Lemma 2)

$$
\left|J_{2}\right| \leqq \frac{2}{\pi} M_{r} \int_{H}\left[\tau_{n}(u)+M\right] K_{n}(u) d u=\frac{2}{\pi} M_{r} \int_{H} \tau_{n}(u) K_{n}(u) d u+o(1) .
$$


Expressing $\tau_{n}(u)$ as a Fejér's integral and interchanging the order of integration, we see that the integral of the right-hand member of (13) is equal to

$$
\frac{2}{\pi^{2}} M_{r} \int_{-\pi}^{\pi} \psi(t) L_{n}(t) d t=\frac{2}{\pi^{2}} M_{r}\left(\int_{-\pi}^{0}+\int_{0}^{\pi}\right)
$$

where $L_{n}(t)$ is given by formula (5). Let $\beta$ be a fixed positive number. We have

$$
\int_{0}^{\pi} \psi(t) L_{n}(t) d t=\int_{0}^{\beta / n} \cdots+\int_{\beta / n}^{\pi}
$$

Let

$$
\Psi(t)=\int_{0}^{t} \psi(u) d u, \quad \Psi(t) \leqq \gamma t \quad(t \geqq 0),
$$

$\boldsymbol{\gamma}$ being a constant (the inequality is implied by the fact of existence of a finite derivative of $\Psi(t)$ at $t=0)$. By (7) the first integral on the right in (14) is $o(n) \Psi(\beta / n)=o(1)$. The second integral is less than

$$
\begin{aligned}
\frac{C}{n} \int_{\beta / n}^{\pi} \psi(t) t^{-2} d t & \leqq \frac{C}{n}\left[\frac{\Psi(\pi)}{\pi^{2}}+2 \int_{\beta / n}^{\pi} \Psi(t) t^{-3} d t\right] \\
& \leqq o(1)+\frac{2 C \gamma}{n} \int_{\beta / n}^{\infty} t^{-2} d t \leqq \epsilon \quad\left(n \geqq n_{0}(\epsilon)\right),
\end{aligned}
$$

where $\epsilon>0$ is arbitrarily small, if only $\beta$ is sufficiently large. An analogous discussion is applied to the integral $\int_{-\pi} \psi(t) L_{n}(t) d t$. It follows that $J_{2} \rightarrow 0$ for every $0<r<1$. Since we may take $r$ as near to 1 as we please, the truth of the lemma follows.

4. Proof of the theorem. Let now $F$ and $G$ denote the sets of points at which, respectively,

$$
\begin{aligned}
& \limsup _{n \rightarrow \infty}\left[s_{n}(x)-f(x)\right]>\underset{n \rightarrow \infty}{\limsup }\left[f(x)-s_{n}(x)\right], \\
& \limsup _{n \rightarrow \infty}\left[s_{n}(x)-f(x)\right]<\underset{n \rightarrow \infty}{\limsup }\left[f(x)-s_{n}(x)\right] .
\end{aligned}
$$

To prove that the set $F$ is of measure 0 , it is enough to show that the set $F_{1}$ of points for which

$$
\limsup _{n \rightarrow \infty}\left[s_{n}(x)-\sigma_{n}(x)\right]>\underset{n \rightarrow \infty}{\lim \sup }\left[\sigma_{n}(x)-s_{n}(x)\right]
$$

is of measure 0 . If it were not, we could find two numbers $N>M>0$ and a set $F_{2} \subset F_{1}$, meas $F_{2}>0$, such that

$$
\underset{n \rightarrow \infty}{\lim \sup }\left[s_{n}(x)-\sigma_{n}(x)\right]>N>M>\limsup _{n \rightarrow \infty}\left[\sigma_{n}(x)-s_{n}(x)\right] .
$$


From the last inequality we conclude the existence of an integer $n_{0}$ and of a set $E \subset F_{2}$, meas $E>0$, such that

$$
\sigma_{n}(x)-s_{n}(x) \leqq M, \quad n>n_{0}, \quad x \in E,
$$

and hence, by Lemma 3, we have

$$
\limsup _{n \rightarrow \infty}\left[s_{n}(x)-\sigma_{n}(x)\right] \leqq M<N
$$

almost everywhere in $E$, contrary to the first of inequalities (16). The theorem is, therefore, established.

5. Additional remarks. (i) Under the hypothesis of our theorem we may prove also that the relation

$$
\tilde{f}(x)=\frac{1}{2}\left[\limsup _{n \rightarrow \infty} \tilde{s}_{n}(x)+\underset{n \rightarrow \infty}{\liminf } \tilde{s}_{n}(x)\right],
$$

where $\tilde{f}(x)$ is the function conjugate to $f(x)$, holds almost everywhere in $(-\pi, \pi)$. The proof is exactly the same as before, except that, instead of (4), we use the formula

$$
\tilde{\sigma}_{n}(x)-\tilde{s}_{n}(x)=\frac{s_{n}^{\prime}(x)}{n+1}=\frac{2}{\pi} \int_{-\pi}^{\pi} s_{n}(u) \sin (n+1)(u-x) K_{n}(u-x) d u .
$$

(ii) It is not difficult to see that the results above may be localized; if we suppose that (2) is satisfied in an interval $(a, b)$, the relations (3) and (17) are true almost everywhere in $(a, b)$. This follows from general localization theorems for trigonometric series.

(iii) The hypothesis that the trigonometric series considered in the theorem is a Fourier-Lebesgue series is superfluous and may be omitted. In fact, inequality (2) implies that the sequence $\left\{\int_{-\pi}^{\pi}\left|s_{n}(x)\right| d x\right\}$ is bounded, and so the series (1) is a Fourier-Stieltjes series. The arguments which we have used in the proof may be, without any difficulty, adapted to this new, slightly more general, case. (See for instance [30].)

\section{ON THE ABSOLUTE CONVERGENCE OF FOURIER SERIES}

1. It has been proved that if $f(x), 0 \leqq x \leqq 2 \pi$, is a periodic function of bounded variation, satisfying a Lipschitz condition of positive order, the Fourier series of $f(x)$ converges absolutely $[28,8]$.

In the same way it is possible to prove the following, more precise, theorem [26].*

* For a similar problem see also O. Szász [23]. 
Theorem 1. If $f(x)$ is of bounded variation and satisfies a Lipschitz condition of order $\alpha$, and if

$$
f(x) \sim \frac{a_{0}}{2}+\sum_{n=1}^{\infty}\left(a_{n} \cos n x+b_{n} \sin n x\right),
$$

then the series

$$
\sum_{n=1}^{\infty} \rho_{n}^{k}, \quad \rho_{n}^{2}=a_{n}^{2}+b_{n}^{2},
$$

converges for every $k>2 /(2+\alpha)$.

The main purpose of this note is to show that the condition imposed on $k$ is the best possible. More precisely, we may state

Theorem 2. For every value $0<\alpha<1$ there exists a function of bounded variation, satisfying a Lipschitz condition of order $\alpha$, and such that the series (2) diverges when $k=2 /(2+\alpha)$.

2. For the sake of completeness we begin by proving Theorem 1 .

Let $N$ be a positive integer and $j=1,2, \cdots, 2 N$. By Parseval's identity,

$$
\begin{aligned}
\int_{0}^{2 \pi} & {\left[f\left(x+\frac{2 \pi j}{2 N}\right)-f\left(x+\frac{2 \pi(j-1)}{2 N}\right)\right]^{2} d x } \\
& =\int_{0}^{2 \pi}\left[f\left(x+\frac{\pi}{2 N}\right)-f\left(x-\frac{\pi}{2 N}\right)\right]^{2} d x=4 \pi \sum_{n=1}^{\infty} \rho_{n}^{2} \sin ^{2} \frac{n \pi}{2 N} .
\end{aligned}
$$

Let $V$ denote the absolute variation of $f(x)$ in the interval $(0,2 \pi)$ and $\omega(\delta)$ the modulus of continuity of $f(x)$, i.e., $\omega(\delta)=\max \left|f\left(x_{1}\right)-f\left(x_{2}\right)\right|$ for $\left|x_{1}-x_{2}\right|$ $\leqq \delta$. In our case $\omega(\delta)=O\left(\delta^{\alpha}\right)$.

For every $x$ we have the inequality

$$
\begin{aligned}
& \sum_{j=1}^{2 N}\left[f\left(x+\frac{2 \pi j}{2 N}\right)-f\left(x+\frac{2 \pi(j-1)}{2 N}\right)\right]^{2} \\
& \quad \leqq \omega\left(\frac{\pi}{N}\right) \sum_{j=1}^{2 N}\left|f\left(x+\frac{2 \pi j}{2 N}\right)-f\left(x+\frac{2 \pi(j-1)}{2 N}\right)\right| \leqq \omega\left(\frac{\pi}{N}\right) V .
\end{aligned}
$$

Integrating this over the range $(0,2 \pi)$, and taking into account (3), we get successively

$$
\begin{gathered}
2 N \sum_{n=1}^{\infty} \rho_{n}^{2} \sin ^{2} \frac{n \pi}{2 N}=O\left(N^{-\alpha}\right), \quad \sum_{n=2^{\nu-1}}^{2^{\nu-1}} \rho_{n}^{2} \sin ^{2} \frac{n \pi}{2 N}=O\left(2^{->(1+\alpha)}\right), \quad N=2^{\nu}, \\
\sum_{n=2^{\nu-1}}^{2^{\nu-1}} \rho_{n}^{2}=O\left(2^{-\nu(1+\alpha)}\right) .
\end{gathered}
$$


We may suppose that $k<2$, the convergence of $\sum \rho_{n}^{2}$ being obvious. Then, by Hölder's inequality,

$$
\sum_{n=2^{y-1}}^{2^{y}-1} \rho_{n}^{k}=O\left(2^{-\nu(1+\alpha) k / 2} \cdot 2^{\nu(1-k / 2)}\right)
$$

It follows that the series

$$
\sum_{n=1}^{\infty} \rho_{n}^{k}=\sum_{\nu=1}^{\infty} \sum_{n=2^{\nu-1}}^{2^{\nu}-1} \rho_{n}^{k}
$$

converges, if only $k>2 /(\alpha+2)$.

3. To prove Theorem 2 we shall consider power series of the form $\sum b_{n} \exp \left(2 \pi i n^{\alpha}\right) z^{n}$, where $0<\alpha<1$ and $b_{n}$ are real and very regularly tend to zero. We shall study these series by means of the following lemmas due to van der Corput.*

LEMMA 1. Let $a(u)$ be a real function of $u,\left|a^{\prime}(u)\right| \leqq 1-\delta$. Then

$$
\left|\sum_{\alpha<\nu \leqq \beta} \exp 2 \pi i a(\nu)-\int_{\alpha}^{\beta} \exp 2 \pi i a(u) d u\right|<A_{\delta} .
$$

where $A_{\delta}$ depends only on $\delta$.

Lemara 2. Let $a^{\prime}(u)$ be positive and decreasing. Then $\dagger$

$$
\left|\int_{\alpha}^{\beta} \exp 2 \pi i a(u) d u\right| \leqq \frac{C}{a^{\prime}(\beta)} .
$$

LEMMA 3. Let $a^{\prime \prime}(u) \leqq-\rho<0$. Then

$$
\left|\int_{\alpha}^{\beta} \exp 2 \pi i a(u) d u\right| \leqq C \rho^{-1 / 2} \text {. }
$$

The following two propositions are (using Abel's transformation) immediate corollaries of Lemmas 1 and 2.

LeMMa 4. If (i) $a(u) \rightarrow \infty$, (ii) $a^{\prime}(u)$ decreases monotonically to zero, (iii) $b_{n} \rightarrow 0$, (iv) $\sum\left|\Delta b_{n}\right|<\infty, \Delta b_{n}=b_{n}-b_{n+1}$, then the series

$$
\sum b_{n} \exp 2 \pi i[a(n)+n \theta]
$$

converges uniformly on every arc $\delta \leqq 0 \leqq 1-\delta$.

* We take these lemmas in the form stated by Hille [10,11]. In [10] several bibliographical references are given.

† See footnote on p. 587. 
LEMMA 5. If (iii) and (iv) in Lemma 4 are replaced respectively by (iii') $b_{n} / a^{\prime}(n) \rightarrow 0$, (iv') $\sum\left|\Delta b_{n}\right| / a^{\prime}(n)<\infty$, then the series (5) converges for every value of $\theta$ although not necessarily uniformly (in fact it converges uniformly over every interval $0 \leqq \theta \leqq 1-\delta$ ).

4. We shall now prove

TheOREM 3. If $0<\alpha<1, \beta>0$, the function

$$
f^{\alpha, \beta}(\theta)=\sum_{n=1}^{\infty} n^{-\beta} \exp 2 \pi i\left(n^{\alpha}+n \theta\right)
$$

which is continuous in every interval $\delta \leqq \theta \leqq 1-\delta$, satisfies the following inequalities:

$$
\begin{aligned}
& f^{\alpha, \beta}(\theta)=\left\{\begin{array}{ll}
O\left(\theta^{-1+\beta /(1-\alpha)}\right), & \alpha+\beta<1 \\
O(\log |\theta|), & \alpha+\beta=1 \\
O(1), & \alpha+\beta>1
\end{array}\right\} \text { as } \theta \rightarrow 0^{+} \\
& f^{\alpha, \beta}(\theta)=\left\{\begin{array}{ll}
O\left(|\theta|^{-(1-\alpha / 2-\beta) /(1-\alpha)}\right), & \frac{1}{2} \alpha+\beta<1 \\
O(1), & \frac{1}{2} \alpha+\beta \geqq 1
\end{array}\right\} \text { as } \theta \rightarrow 0^{-} .
\end{aligned}
$$

Let

$$
S_{n}^{\alpha, \beta}(\theta)=\sum_{\nu=1}^{n} \nu^{-\beta} \exp 2 \pi i\left(\nu^{\alpha}+\nu \theta\right) .
$$

Put $a(u)=u^{\alpha}+u \theta$ and assume $|\theta| \leqq \frac{1}{2}$. From Lemma 2 it follows that

$$
S_{n}^{\alpha, 0}(\theta)=\int_{1}^{n} \exp 2 \pi i a(u) d u+O(1)
$$

and, by Lemma $3, *$

Hence

$$
\left|\int_{1}^{n} \exp 2 \pi i a(u) d u\right| \leqq A n^{1-\alpha / 2} .
$$

$$
\left|S_{n}^{\alpha, 0}(\theta)\right| \leqq A n^{1-\alpha / 2}, \quad|\theta| \leqq \frac{1}{2} .
$$

For subsequent discussion we need more precise estimates of $S_{n}^{\alpha, 0}(\theta)$. To obtain them the cases $0<\theta \leqq \frac{1}{2},-\frac{1}{2} \leqq \theta<0$ have to be treated separately. In the first case we have, by Lemma 2,

$$
\left|\int_{1}^{n} \exp 2 \pi i a(u) d u\right| \leqq \frac{A}{\alpha n^{\alpha-1}+\theta} \leqq \frac{A}{\theta},
$$

* We use $A$ as a general notation of a constant which does not depend on $\theta$. 
whence

$$
\left.\begin{array}{l}
\left|S_{n}^{\alpha, 0}(\theta)\right| \leqq A n^{1-\alpha} \\
\left|S_{n}^{\alpha, 0}(\theta)\right| \leqq \frac{A}{\theta}
\end{array}\right\} 0<\theta \leqq \frac{1}{2} .
$$

In the second case we put $t=-\theta$,

$$
\begin{gathered}
a(u)=u^{\alpha}-t u, \quad a^{\prime}(u)=\alpha u^{\alpha-1}-t, \quad 0<t \leqq \frac{1}{2}, \\
N_{1}=\left[\left(\frac{2 t}{\alpha}\right)^{-1 /(1-\alpha)}\right], \quad N_{2}=\left[\left(\frac{t}{2 \alpha}\right)^{-1 /(1-\alpha)}\right] .
\end{gathered}
$$

Now if $n \leqq N_{1}$, again by Lemma 2,

$$
\left|\int_{1}^{n} \exp 2 \pi i a(u) d u\right| \leqq \frac{A}{\alpha n^{\alpha-1}-t} \leqq A n^{1-\alpha},
$$

and again

$$
\left|S_{n}^{\alpha, 0}(\theta)\right| \leqq A n^{1-\alpha}, \quad n \leqq N_{1}
$$

By (8),

$$
\left|S_{n}^{\alpha, 0}(\theta)\right| \leqq A n^{1-\alpha / 2} \leqq A|\theta|^{-1-\alpha /[2(1-\alpha)]}, \quad N_{1}<n \leqq N_{2} .
$$

Finally, when $n>N_{2}$, we write

$$
\int_{1}^{n} \exp 2 \pi i\left(u^{\alpha}-t u\right) d u=\int_{1}^{N_{2}} \cdots+\int_{N_{2}}^{n} \cdots .
$$

The first integral on the right gives the same contribution as (14) while the second integral can be estimated by Lemma 2 (with an obvious modification). Thus we get

$$
\left|\int_{N_{2}}^{n} \exp 2 \pi i\left(u^{\alpha}-t u\right) d u\right| \leqq \frac{A}{t-\alpha N_{2}^{\alpha-1}} \leqq \frac{A}{t}
$$

and

$$
\left|S_{n}^{\alpha, 0}(\theta)\right| \leqq|\theta|^{-1-\alpha /[2(1-\alpha)]}, \quad n>N_{2} .
$$

Now, by Abel's partial summation,

$$
S_{n}^{\alpha, \beta}(\theta)=\sum_{\nu=1}^{n-1} S_{\nu}^{\alpha, 0}(\theta) \Delta \nu^{-\beta}+n^{-\beta} S_{n}^{\alpha, 0}(\theta)
$$


For any fixed value of $\theta \neq 0,|\theta| \leqq \frac{1}{2}$, the second term of the right-hand member tends to 0 as $n \rightarrow \infty$. Hence

$$
f^{\alpha, \beta}(\theta)=\lim _{n \rightarrow \infty} S_{n}^{\alpha, \beta}(\theta)=\sum_{\nu=1}^{\infty} \Delta \nu^{-\beta} S_{\nu}^{\alpha, 0}(\theta), \quad 0<|\theta| \leqq \frac{1}{2} .
$$

We write

By (9) and (13)

$$
f^{\alpha, \beta}(\theta)=\sum_{\nu=1}^{N_{1}} \Delta \nu^{-\beta} S_{\nu}^{\alpha, 0}(\theta)+\sum_{\nu=N_{1}+1}^{\infty} \Delta \nu^{-\beta} S_{\nu}^{\alpha, 0}(\theta) \equiv P+Q .
$$

Hence, for $0<|\theta| \leqq \frac{1}{2}$,

$$
|P| \leqq A \sum_{\nu=1}^{N_{1}} \nu^{-1-\beta, 1-\alpha}=A \sum_{\nu=1}^{N_{1}} \nu^{-\alpha-\beta} .
$$

$$
P= \begin{cases}O\left(N_{1}^{1-\alpha-\beta}\right)=O(|\theta|-1+\beta /(1-\alpha)), & \text { if } \alpha+\beta<1, \\ O\left(\log N_{1}\right)=O(\log |\theta|), & \text { if } \alpha+\beta=1, \\ O(1), & \text { if } \alpha+\beta>1 .\end{cases}
$$

At this juncture we have again to distinguish between the cases $0<\theta \leqq \frac{1}{2}$ and $-\frac{1}{2} \leqq \theta<0$. In the first case we apply (10) which gives

$$
Q=O\left(\frac{1}{\theta} \sum_{\nu=N_{1}+1}^{\infty} \nu^{-1-\beta}\right)=O\left(\theta^{-1} N_{1}^{-\beta}\right)=O\left(\theta^{-1+\beta /(1-\alpha)}\right) .
$$

Being combined with the estimates above for $P$ this furnishes the proof of the first part of Theorem 3 .

When $-1 \leqq \theta<0$ we write

$$
Q=\sum_{n=N_{1}+1}^{\infty} \cdots=\sum_{n=N_{1}+1}^{N_{2}} \cdots+\sum_{y=N_{2}+1}^{\infty} \cdots \equiv R+S,
$$

and apply (14) and (15). This yields

$$
|R| \leqq A \sum_{n=N_{1}+1}^{N_{2}} \nu^{-1-\beta_{\nu} 1-\alpha / 2}=A \sum_{\nu=N_{1}+1}^{N_{2}} \nu^{-\beta-\alpha / 2} .
$$

It follows that

$$
R= \begin{cases}O\left(N_{2}^{1-\beta-\alpha / 2}\right)=O(|\theta|-(1-\beta-\alpha / \mathbf{z}) /(1-\alpha)), & \text { if } \beta+\frac{\alpha}{2}<1 \\ O\left(\log \frac{N_{2}}{N_{1}}\right)=O(1), & \text { if } \beta+\frac{\alpha}{2}=1 \\ o(1), & \text { if } \beta+\frac{\alpha}{2}>1\end{cases}
$$


Finally, $S$ is readily estimated by using (15) which gives

$$
\begin{aligned}
S & =O\left(\sum_{\nu=N_{2}+1}^{\infty} \nu^{-1-\beta}|\theta|^{-1-\alpha /[2(1-\alpha)]}\right) \\
& =O\left(|\theta|^{-1-\alpha /[2(1-\alpha)]} N_{2}^{-\beta}\right)=O\left(|\theta|^{-(1-\beta-\alpha / 2) /(1-\alpha)}\right) .
\end{aligned}
$$

On combining these results we obtain a proof of the second part of Theorem 3.

Theorem 3 shows that the behavior of $f^{\alpha, \beta}(\theta)$ in the neighborhood of $\theta=0$ is different for $\theta \rightarrow 0^{+}$and for $\theta \rightarrow 0^{-}$. In the interval $0<\theta \leqq \frac{1}{2}$ the function $f^{\alpha, \beta}(\theta)$ is always integrable. In the interval $-\frac{1}{2} \leqq \theta<0$ we are sure of integrability only if $\beta>\alpha / 2$. If $\beta=\alpha / 2$ we get only $f^{\alpha, \beta}(\theta)=O\left(|\theta|^{-1}\right)$ and the function is probably not integrable.* It will be integrable if we introduce additional logarithms, as is shown in the next

TheOREM 4. The sum of the series

$$
\sum_{n=2}^{\infty} n^{-\alpha / 2}(\log n)^{-\gamma} \exp 2 \pi i\left(n^{\alpha}+n \theta\right), \quad \gamma>1,
$$

is $O\left(|\theta|^{-1} \log ^{-\gamma}(1 /|\theta|)\right)$, and, consequently, the series is the Fourier series of its sum.

Although this theorem is important for our purposes, the proof need not be gone into, as it is essentially the same as that of Theorem 3. $\dagger$

5. We now prove

THEOREM 5. If $1 \leqq \alpha / 2+\beta \leqq 2,0<\alpha<1, \beta>0$, the function $f^{\alpha, \beta}(\theta)$ satisfies a Lipschitz condition of order $\alpha / 2+\beta-1$.

The case $\alpha / 2+\beta=1$ is contained in Theorem 3 and the other extreme case is a corollary of it. If $1<\alpha / 2+\beta<2$ it follows from Lemma 5 that the series is everywhere convergent. Using (16) we have, with $N=[1 /|h|]$,

$$
\begin{aligned}
\left|f^{\alpha, \beta}(\theta+h)-f^{\alpha, \beta}(\theta)\right| & \leqq \sum_{\nu=1}^{\infty} \Delta_{\nu}^{-\beta}\left|S_{\nu}^{\alpha, 0}(\theta+h)-S_{\nu}^{\alpha, 0}(\theta)\right| \\
& =\sum_{n=1}^{N} \cdots+\sum_{n=N+1}^{\infty} \cdots \equiv P+Q .
\end{aligned}
$$

From (8) it follows that

- It is certainly not integrable if $\beta<\alpha / 2$, for othertise the series $\Sigma n^{-1-\beta} \exp 2 \pi i\left(n^{\alpha}+n \theta\right)$ would be the Fourier series of a function of bounded variation (indeed absolutely continuous) satisfying a Lipschitz condition of order $\alpha / 2+\beta$ (see Theorem 5) and, by Theorem 1 , its exponent of convergence would be $\leqq 2 /(\alpha / 2+\beta+2)$, which is easily seen to be impossible. It is, however, obvious that for any $\alpha, \beta>0$, the series (6) are Fourier-Riemann series.

$\dagger$ The same argument gives a more general result concerning the functions we obtain by introducing logarithms into the denominator of the series (6). 


$$
\begin{aligned}
|Q| & \leqq \sum_{n=N+1}^{\infty} \Delta \nu^{-\beta}\left(\left|S_{\nu}^{\alpha, 0}(\theta+h)\right|+\left|S_{\nu}^{\alpha, 0}(\theta)\right|\right)=O\left(\sum_{,-N+1}^{\infty} \nu^{-\beta-1} \nu^{1-\alpha / 2}\right) \\
& =O\left(\mid h^{\alpha / 2+\beta-1}\right) .
\end{aligned}
$$

On the other hand we have

$$
|P| \leqq|h| \sum_{\nu=1}^{N} \Delta \nu^{-\beta} \max \left|\left(S_{\nu}^{\alpha, 0}\right)^{\prime}\right| .
$$

By the well known theorem of S. Bernstein, if $T_{n}(\theta)$ is any trigonometric polynomial of order $n$, and if $\left|T_{n}(\theta)\right| \leqq M$, then $\left|T_{n}^{\prime}(\theta)\right| \leqq M n$. In view of (8) this yields at once

$$
\left|\frac{d}{d \theta} S_{,}^{\alpha, 0}(\theta)\right| \leqq A \nu^{2-\alpha / 2},
$$

whence

$$
|P|=O\left(|h| \sum_{\nu=1}^{N} \nu^{-1-\beta_{\nu} 2-\alpha / 2}\right)=O\left(|h| N^{2-\alpha / 2-\beta}\right)=O\left(|h|^{\alpha / 2+\beta-1}\right) .
$$

Theorem $\mathbf{5}$ is thus proved.

Theorem 6. If $1 \leqq \alpha / 2+\beta \leqq 2,0<\alpha<1, \beta>0, \gamma>0$, then the sum of the series

$$
f^{\alpha, \beta, \gamma}(\theta)=\sum_{n=2}^{\infty} n^{-\beta}(\log n)^{-\gamma} \exp 2 \pi i\left(n^{\alpha}+n \theta\right)
$$

satisfies a Lipschitz condition of order $\frac{1}{2} \alpha+\beta-1$.

The proof is the same as in the case of Theorem $5 .^{*}$

THEOREM 7. The series

$$
\sum_{n=2}^{\infty} n^{-1-\alpha / 2}(\log n)^{-\gamma} \exp 2 \pi i\left(n^{\alpha}+n \theta\right), 0<\alpha<1, \gamma>1,
$$

is the Fourier series of a function of bounded variation satisfying a Lipschitz condition of order $\alpha$, and if $\gamma$ is sufficiently near to 1 , its coefficients $c_{n}$ have the property that $\sum\left|c_{n}\right|^{k}$ diverges for $k=2 /(\alpha+2)$.

This follows from Theorems 4 and 6. Theorem 2 now follows from Theorem 7 .

* We may also deduce Theorem 6 from Theorem 5 if we take into account that (18) is a "Faltung" of (6) and $\Sigma(\log n)^{-\gamma} \cos 2 \pi n \theta$ which is a Fourier-Lebesgue series for every $\gamma>0$. It is easy to see that the modulus of continuity of (6) will be preserved. 


\section{ON A THEOREM OF FeJER AND RIESZ}

1. The following result has been obtained by Fejér and Riesz [4].

ThEOREM 1. Every analytic function $f(z)$ regular for $|z| \leqq 1$ satisfies the inequality

$$
\int_{D}|f(z)| p|d z| \leqq \frac{1}{2} \int_{C}|f(z)| p|d z|
$$

where $C$ denotes the circle $|z|=1$ and $D$ is its arbitrary diameter.

It is well known that it suffices to prove the inequality $\left(1_{p}\right)$ for any special value of $p$; the general result then follows by a familiar argument.* Fejer and Riesz started with the case $p=2$. An alternative proof of $\left(1_{p}\right)$ which is given below begins with $p=1$. This proof is based on the following

LEMma. Let $u(z)$ and $v(z)$ be conjugate, not necessarily real, $\dagger$ harmonic functions such that $v(0)=0$ and that $f(z)=u(z)+i v(z)$ is regular for $|z| \leqq 1$. Then, with the same notations as before,

$$
\int_{D}|v(z)||d z| \leqq \int_{D}\left|\frac{v(z)}{z}\right||d z| \leqq \frac{1}{2} \int_{C}|u(z)||d z| .
$$

On setting

$$
Q_{r}(t)=\sum_{n=1}^{\infty} r^{n} \sin n t=\frac{r \sin t}{1-2 r \cos t+r^{2}}, \quad z=r e^{i \theta},
$$

we have

$$
v\left(r e^{i \theta}\right)=\frac{1}{\pi} \int_{0}^{2 \pi} u\left(e^{i t}\right) Q_{r}(t-\theta) d t .
$$

Without loss of generality we may assume that the diameter $D$ is the segment $(-1,1)$ of the $X$-axis. Then

$$
\int_{D}\left|\frac{v(z)}{z}\right||d z|=\int_{0}^{1}[|v(r)|+|v(-r)|] r^{-1} d r \leqq M \int_{0}^{2 \pi}\left|u\left(e^{i t}\right)\right| d t,
$$

* It is well known that the condition of $f(z)$ being regular on $C$ is not necessary and may be replaced by less stringent conditions. In the proof of Theorem 2 below we shall use the inequality $\left(1_{p}\right)$ under the assumption that $\Re(f)$ is continuous for $|z| \leqq 1$.

t A complex harmonic function $v(z)=v_{1}(z)+i v_{2}(z)$ is said to be conjugate to $u_{1}(z)+i u_{2}(z)$ if $\nabla_{1}(z)$ is conjugate to $u_{1}(z)$, and $v_{2}(z)$ to $u_{2}(z)$. 
where $M$ is the upper bound, with respect to $\alpha$, of

$$
\frac{1}{\pi} \int_{0}^{1}\left[\left|Q_{r}(\alpha)\right|+\left|Q_{r}(\alpha+\pi)\right|\right] r^{-1} d r .
$$

Suppose, as we may, that $0<\alpha<\pi$. On setting $\sin \alpha=h, \cos \alpha=k$, we see that the last integral is equal to

$$
\frac{h}{\pi}\left[\int_{0}^{1} \frac{d r}{(r-k)^{2}+h^{2}}+\int_{0}^{1} \frac{d r}{(r+k)^{2}+h^{2}}\right]=\frac{1}{2} .
$$

Our lemma is thus established. $\dagger$

Now we notice that if $g(z)$ is analytic, the function $-i g(z)$ is conjugate to $g(z)$. Consequently, applying our lemma to the functions $u(z)=z f(z)$ and $v(z)=-i z f(z)$, we get the inequality $\left(1_{1}\right)$ and, hence, the whole Theorem 1 .

2. We nów prove

THEOREM 2. Let $f(z)=u(z)+i v(z)$ be regular for $|z| \leqq 1$, where $u$ and $v$ are real and $v(0)=0$. There exists a constant $A_{p}$ depending only on $p$, and uniformly bounded in every interval $1 \leqq p \leqq p_{0}$, such that

$$
\int_{D}|v(z)| p|d z| \leqq A_{p}^{p} \int_{C}|u(z)| p|d z|, \quad p \geqq 1 .
$$

A preliminary remark is worth making. It has been proved by M. Riesz [22] that for any $p>1$ we have

$$
\int_{0}^{2 \pi}\left|v\left(e^{i \theta}\right)\right|^{p} d \theta \leqq M_{p} \int_{0}^{2 \pi}\left|u\left(e^{i \theta}\right)\right|^{p} d \theta
$$

where $M_{p}$ depends only on $p$, and so

$$
\begin{aligned}
\int_{D}|v(z)|^{p}|d z| & \leqq \int_{D}|f(z)| p|d z| \leqq \frac{1}{2} \int_{C}|f(z)| p|d z| \\
& \leqq 2^{p-1} \int_{C}\left[|u(z)|^{p}+|v(z)| p\right]|d z| \\
& \leqq 2^{p-1}\left(M_{p}+1\right) \int_{C}|u(t)|^{p}|d z| .
\end{aligned}
$$

$\dagger$ The constant $\frac{1}{2}$ in (2) cannot be improved for, otherwise, we could improve the inequality $\left(1_{p}\right)$, which is known to be impossible (Fejer and Riesz, loc. cit.). Another example is given by the pair of conjugate functions $u(R z)$ and $v(R z)$, where

$$
u(z)=P_{r}(\theta)=\frac{1}{2} \frac{1-r^{2}}{1-2 r \cos \theta+r^{2}}, \quad v(z)=Q_{r}(\theta),
$$

and $R$ is sufficiently near to 1 . 
However we cannot put $A_{p}^{p}=2^{p-1}\left(M_{p}+1\right)$, since $M_{p}$ is known to be unbounded in the neighborhood of $p=1$, so that Theorem 2 is not a consequence of $\left(1_{p}\right)$ and of $M$. Riesz's theorems on conjugate functions, although every single inequality $\left(3_{p}\right)$, for $p>1$, is such a consequence.

Assume again for simplicity that $D$ is the interval $(-1,1)$ of the $X$-axis. To any continuous function $u\left(e^{i \theta}\right)$ defined on $|z|=1$, there corresponds a function $v(z)=T\{u\}$, conjugate to the Poisson integral of $u\left(e^{i \theta}\right)$, defined for $-1<r<1$. The functional $v=T\{u\}$ is. additive and the inequality $\left(3_{p}\right)$ is certainly true for $p=1$ and $p=p_{0}$. By a theorem of M. Riesz [21], the upper bound (with respect to all continuous functions) of the ratio

$$
\left(\int_{-1}^{1}|v(r)|^{p} d r\right)^{1 / p} /\left(\int_{0}^{2 \pi}\left|u\left(e^{i \theta}\right)\right|^{p} d \theta\right)^{1 / p}
$$

is a convex function of $1 / p, p \geqq 1$. Hence, if $A_{p}$ denotes the smallest possible value for which $\left(3_{p}\right)$ is true and if $1 \leqq p \leqq p_{0}$, the number $A_{p}$ does not exceed $\max \left(A_{1}, A_{p_{0}}\right)$.

TheOREM 3. Under the conditions of Theorem 2 we also have

$$
\int_{D}\left|\frac{v(z)}{z}\right|^{p}|d z| \leqq A_{p}^{p} \int_{C}|u(z)|^{p}|d z|
$$

where $A_{p}$ is a constant analogous to, but not necessarily the same as, the constant $A_{p}$ of Theorem 2.

In fact, if $a_{0}=f(0)$, we find, arguing as above, that

$$
\begin{aligned}
\int_{D}\left|\frac{v(z)}{z}\right|^{p}|d z| & \leqq \int_{D}\left|\frac{f(z)-a_{0}}{z}\right|^{p}|d z| \\
& \leqq 2^{p-1}\left(M_{p}+1\right) \int_{C}\left|u(z)-a_{0}\right|^{p}|d z| \\
& \leqq A_{p}^{* p} \int_{C}|u(z)| p|d z|,
\end{aligned}
$$

since

$$
\left|a_{0}\right|^{p}=|f(0)|^{p} \leqq\left(\frac{1}{2 \pi} \int_{0}^{2 \pi}\left|u\left(e^{i \theta}\right)\right| d \theta\right)^{p} \leqq \frac{1}{2 \pi} \int_{0}^{2 \pi}\left|u\left(e^{i \theta}\right)\right|^{p} d \theta .
$$

The rest of the proof is the same.

3. Additional remarks. (i) The function $u(z)=P_{r}(\theta)$ shows that Theorem 2 is false if in the left-hand member of the inequality (3) we replace $v$ by $u$, but, of course, the new inequality is true if $1+\epsilon \leqq p \leqq p_{0}$, for every $\epsilon>0$.

(ii) Let $u(z)$ be real and harmonic for $|z|<1$. Applying the Lemma to the 
conjugate functions $\partial u / \partial \theta$ and $-r \partial u / \partial r$ we obtain the following result: If a function $u\left(e^{i \theta}\right)$ is of bounded variation, the corresponding harmonic function defined by Poisson's integral is of (uniformly) bounded variation on any radius (cf. Prasad [19]. where a more general result is proved).

(iii) Theorem 2 is probably false for any $0<p<1$. It is certainly false e.g. for $p=\frac{1}{2}$, as the example of conjugate functions $d P_{r}(\theta) / d \theta$ and $d Q_{r}(\theta) / d \theta$ shows (see footnote on page 600).

\section{ON A THEOREM ON CONJUGATE FUNCTIONS}

1. The following is one of the several definitions of an integral given by Denjoy [2].

A function $f(x)$ defined for $a \leqq x \leqq b$ and continued outside $(a, b)$ by the condition of periodicity is said to be integrable $B$ on $(a, b)$ if, for an arbitrary subdivision $a=a_{0}<a_{1}<a_{2}<\cdots<a_{n}=b$ and arbitrary set of values $\xi_{i}$, $a_{i-1} \leqq \xi_{i} \leqq a_{i}$, the expression

$$
J(f ; t)=\sum_{i=1}^{n} f\left(\xi_{i}+t\right)\left(a_{i}-a_{i-1}\right)
$$

tends in measure to a limit $J$, when $\max \left(a_{i}-a_{i-1}\right) \rightarrow 0 . \dagger J$ is then called the value of the integral of $f$ over $(a, b)$.

It is not difficult to grasp the meaning of the above definition. Instead of one (periodic) function $f(x)$, we consider the whole family $f_{t}(x)$ derived from $f(x)$ by translating the argument $x$ by $t$ and construct for each of them the Riemannian approximating sums. Even if the function $f(x)$ (and, consequently, any $\left.f_{t}(x)\right)$ is not integrable $R$, it may happen that "on the whole" the sums $J(f ; t)$ are near to a number $J$, and the nearer, the smaller max $\left(a_{i}-a_{i-1}\right)$ is. Thus, the integral $B$ is what may be called "Riemann's integral in measure."

This definition has found a rather unexpected application in the theory of trigonometric series by the following theorem of Kolmogoroff [14]:

Theorem A. If $f(x)$ is integrable $L$ and

$$
f(x) \sim \frac{a_{0}}{2}+\sum_{n=1}^{\infty}\left(a_{n} \cos n x+b_{n} \sin n x\right)
$$

the (generalized) sum $\tilde{f}(x)$ of the conjugate series

$$
\sum_{n=1}^{\infty}\left(a_{n} \sin n x-b_{n} \sin n x\right)
$$

is integrable B, and, moreover, (3) is the Fourier (-Denjoy) series of $\tilde{f}(x)$.

$\dagger$ In other words, for every $\epsilon>0$ there exists a $\delta=\delta(\epsilon)$, such that, if only $\max \left(a_{i}-a_{i-1}\right)<\delta$, the measure of the set of values of $t$ for which $|J-J(f ; t)|>\epsilon$ is less than $\epsilon$. 
Kolmogoroff's proof is based on an inequality concerning the measure of the set of points for which $|\tilde{f}(x)| \geqq R$. As the proofs of this inequality, so far published [13,25], are not simple, an alternative proof of Theorem A would be, perhaps, of some interest. The proof given below uses a theorem (Theorem C) also due to Kolmogoroff, which may be considered now as fairly simple (cf. Hardy [5]).

2. We begin by proving the following theorem of Denjoy $[2,1]$ (which is not necessary for the proof of Theorem $A$ ).

THEOREM B. If $f(x)$ is integrable $L$ in $(a, b)$ it is also integrable $B$ and both definitions give the same value of the integral. $\dagger$

Let

$$
J=(L) \int_{a}^{b} f(x) d x, \quad J^{*}=(L) \int_{a}^{b}|f(x)| d x .
$$

Integrating (1) we get

$$
\int_{a}^{b}|J(f ; t)| d t \leqq \sum_{i=1}^{n}\left(a_{i}-a_{i-1}\right) \int_{a}^{b}\left|f\left(\xi_{i}+t\right)\right| d t=(b-a) J^{*} .
$$

Suppose that $J^{*}<\epsilon^{2} /(3(b-a))$. Then the left-hand member in (4) does not exceed $\epsilon^{2} / 3$ and the measure of the set of values of $t$ for which $|J(f ; t)|>\epsilon / 3$ does not exceed $\epsilon$. In the general case we put $f=f_{1}+f_{2}$ and introduce the integrals $J_{1}, J_{1}{ }^{*}, J_{2}, J_{2}{ }^{*}$, analogous to $J, J^{*}$. We may suppose that $f_{1}$ is continuous and that $J_{2}{ }^{*} \leqq \epsilon^{2} /(3(b-a))$. Then $\left|J\left(f_{2} ; t\right)\right| \leqq \epsilon / 3$ except in a set of measure $\leqq \epsilon$. On the other hand, if $\max \left(a_{i}-a_{i-1}\right)$ is sufficiently small, we have for every $t$ the inequality $\left|J\left(f_{1} ; t\right)-J_{1}\right|<\epsilon / 3$ and so (assuming as we may, that $\epsilon<b-a)$

$$
\begin{aligned}
|J(f ; t)-J| & \leqq\left|J\left(f_{1} ; t\right)-J_{1}\right|+\left|J\left(f_{2} ; t\right)\right|+\left|J_{2}\right| \\
& \leqq \epsilon / 3+\epsilon^{2} /(3(b-a))+\epsilon^{2} /(3(b-a))<\epsilon
\end{aligned}
$$

except in a set of measure $\leqq \epsilon$.

3. The theorem which we will use in the proof of Theorem A and which we take for granted is as follows. then

Theorem C. If $f(x)$ is integrable L over $(0,2 \pi)$, and $\tilde{f}(x)$ is conjugate to $f$,

$$
\left(\int_{0}^{2 \pi}|\tilde{f}(x)|^{1-\epsilon} d x\right)^{1 /(1-\epsilon)} \leqq A_{\epsilon} \int_{0}^{2 \pi}|f(x)| d x,
$$

where $A_{\epsilon}$ is a constant depending only on $\epsilon>0$.

$\dagger$ The proof given in the text is due to Dr. S. Saks. 
Now it is obvious that if we replace in (1) $f$ by $\tilde{f}$, we obtain the function $J(\tilde{f} ; t)$ conjugate to $J(f ; t)$. Hence, from (5), with $\epsilon=\frac{1}{2}$ we get

$$
\int_{0}^{2 \pi}|J(\tilde{f} ; t)|^{1 / 2} d t \leqq A_{1 / 2}^{1 / 2}\left(\int_{0}^{2 \pi}|f(t)| d t\right)^{1 / 2} .
$$

Suppose first that the right-hand member of (6) does not exceed $\epsilon^{3 / 2}$. Then the set of values of $t$ for which $J(\tilde{f} ; t)$ exceeds $\epsilon$ is less than $\epsilon$. In the general case we put again $f=f_{1}+f_{2}$, where $f_{1}$ has a continuous derivative (so that $\tilde{f}_{1}$ is continuous) and the integral of $\left|f_{2}\right|$ is small. In the equality $J(\tilde{f} ; t)=J\left(\tilde{f}_{1} ; t\right)$ $+J\left(\tilde{f}_{2} ; t\right)$ the term $J\left(\tilde{f}_{1} ; t\right)$ is small for every $t$, provided that $\max \left(a_{i}-a_{i-1}\right)$ is small (the Fourier series of $\tilde{f}_{1}$ has no constant term) and $J\left(\tilde{f}_{2} ; t\right)$ is small, except in a set of small measure. This shows that $\tilde{f}$ is integrable $B$ and the value of the integral is 0 , as was to be expected.

4. To prove the second part of Theorem A, we have to show that the products $f(x) \cos k x$ and $f(x) \sin k x$ are integrable $B$ and that the corresponding integrals are $-\pi b_{k}, \pi a_{k}, k=1,2, \cdots$. We may suppose that $a_{0}=a_{1}=$ $b_{1}=\cdots=a_{k}=b_{k}=0$. Then it is not difficult to verify that the conjugate functions of $f(x) \cos k x, f(x) \sin k x$, are $\tilde{f}(x) \cos k x, \tilde{f}(x) \sin k x$ respectively. Hence the products $\tilde{f}(x) \cos k x, \tilde{f}(x)$ sin $k x$ are integrable $B$ and their integrals over $(0,2 \pi)$ vanish.

\section{ON AN EXTREME CASE IN THE THEORY OF FRACTIONAL INTEGRALS}

1. Hardy and Littlewood have proved [7] that if $f(x)$ belongs to $L^{p}(p>1)$ in an interval $(a, b)$, where $-\infty<a<b \leqq \infty$, then the function $f_{\alpha}(x)$, the fractional integral of order $\alpha$ of $f(x)$, belongs to $L^{q}$, provided that

$$
1 / p-1 / q=\alpha, \quad 0<\alpha<1 / p, \quad p>1 .
$$

As may be shown by very simple examples [7], this theorem is no longer true when $p=1$. The main purpose of this note is to find a substitute theorem for this case and to give some indications concerning the case $\alpha=1 / p$. Since these theorems have some applications in the theory of Fourier series, Weyl's definition of fractional integral [27] will be more convenient for us and we shall use it throughout, instead of the familiar Riemann-Liouville definition. According to Weyl's definition

$$
f_{\alpha}(x)=\frac{1}{\Gamma(\alpha)} \int_{-\infty}^{x}(x-t)^{\alpha-1} f(t) d t, \quad 0<\alpha<1,
$$

where the integrable function $f$ has the period $2 \pi$ and the constant coefficient of its Fourier series vanishes. The latter condition will be tacitly assumed throughout this paper, wherever it is necessary. 
The arguments will be based on the theorem just mentioned, which it will be necessary for our purposes to state in its complete form.

Theorem 1 (Hardy-Littlewood). If $f(x) \subset L^{p}, p>1$, in the interval $(0,2 \pi)$ and if the relations (1) are satisfied, then

$$
\mathfrak{M}_{q}\left(f_{\alpha}\right)<M \mathfrak{M}_{p}(f)
$$

with

$$
M=\mu q^{q^{\prime} / p^{\prime}},
$$

where $\mu$ is an absolute constant. $\dagger$

2. We begin by proving the following

THEOREM 1. Suppose that $f \subset L^{r}, r>1$, and that $\mathfrak{M}_{r}(f) \leqq 1$. Then there exist two constants $\lambda>0$ and $\Lambda$ independent of $f$, such that

$$
\int_{0}^{2 \pi} \exp \lambda\left|f_{1 / r}(x)\right| r^{\prime} d x<\Lambda .
$$

This result shows that the function $f_{1 / r}(x)$, which by the theorem of Hardy and Littlewood is integrable in any power, is integrable exponentially.

Put in (2)

$$
\begin{aligned}
\alpha & =1 / r, \quad p=\frac{r k}{r+k-1}<r, \\
p^{\prime} & =\frac{r k}{(r-1)(k-1)}, \quad q=r^{\prime} k>p, \quad k=2,3, \cdots .
\end{aligned}
$$

Then, since $f \subset L^{p}$, and since $\mathfrak{M}_{p}(f)$ is an increasing function of $p$ we deduce from (2), (3) that

where

$$
\begin{aligned}
\mathfrak{M}_{r^{\prime} k}\left(f_{1 / r}\right) & \leqq D_{k} \mathfrak{M}_{p}(f) \\
& \leqq D_{k} \mathfrak{M}_{r}(f) \leqq D_{k},
\end{aligned}
$$

$$
D_{k}=\mu q^{q^{\prime} / p^{\prime}}=\mu\left(r^{\prime} k\right)^{(k-1) /\left(r^{\prime} k-1\right)}<\mu\left(r^{\prime} k\right)^{1 / r^{\prime}} .
$$

Raising the inequality (5) to the power $r^{\prime} k$, multiplying it by $\lambda^{k} / k !$, and summing from $k=2$ on, we get, by Stirling's formula,

† We use the familiar notation

$$
\mathfrak{M}_{r}(\phi)=\left(\frac{1}{2 \pi} \int_{0}^{2 \pi}|\phi| r d x\right)^{1 / r}, \quad r^{\prime}=\frac{r}{(r-1)} .
$$

The numerical value of $\mu$ in (3) is irrelevant for our purposes. When the Riemann-Liouville definition is used (in the interval $(0, \infty)$ ) we may put e.g. $\mu=\max 1 / \Gamma(1+\alpha)$. For the definition adopted in this paper the value of $\mu$ ten times as large will certainly be sufficient. 


$$
\begin{aligned}
\sum_{k=2}^{\infty} \frac{\lambda^{k}}{k !} \int_{0}^{2 \pi}\left|f_{1 / r}\right|^{r^{\prime} k} d x & \leqq 2 \pi \sum_{k=2}^{\infty} \frac{\left(\mu^{r^{\prime}} r^{\prime} \lambda\right)^{k} k^{k}}{k !} \leqq C \sum_{k=2}^{\infty}\left(\mu^{r^{\prime}} r^{\prime} \lambda e\right)^{k} \\
& <\frac{C}{1-\mu^{r^{\prime}} r^{\prime} \lambda e}=B,
\end{aligned}
$$

where $C$ is an absolute constant and $\lambda$ is assumed to be so small that $\mu^{r^{\prime}} r^{\prime} \lambda e<1$. Let $\psi(x)=e^{x}-1-x$. Noticing that for $x \geqq 0, e^{x} \leqq 2 \psi(x)+C$ (see footnote on page 587), and taking into account only the extreme terms of the inequality (6), we see that, with a modified value of $B$, we may replace in the first of them the lower limit of summation by 0 , and this is just the inequality (4).

3. If we put (which we have no right to do) in the second relation (1) $p=1$, we should obtain $q=1 /(1-\alpha)$. But the theorem is false for $p=1$; to state the correct form of this extreme case we introduce the class $L^{1, k}$ of functions $f$ such that $|f|\left(\log ^{+}|f|\right)^{k}$ is integrable. We have then

THeOREM 2. If $f \subset L^{1,1-\alpha}, 0<\alpha<1$, then $f_{\alpha} \subset L^{\beta}, \beta=1 /(1-\alpha)$, and

$$
\mathfrak{M}_{\beta}\left(f_{\alpha}\right) \leqq M \int_{0}^{2 \pi}|f|\left(\log ^{+}|f|\right)^{1-\alpha} d x+N,
$$

where the constants $M$ and $N$ do not depend on $f . \dagger$

Given any integrable function $\phi(x), 0 \leqq x \leqq 2 \pi$, we shall denote by $\sigma_{n}[\phi]$ the first arithmetical means of the Fourier series of $\phi$. It is well known that the two inequalities

$$
\mathfrak{M}_{r}(\phi) \leqq A, \quad \mathfrak{M}\left(\sigma_{n}[\phi]\right) \leqq A,
$$

where $A$ is a constant and $r \geqq 1$, are equivalent. Therefore, if we wish to prove that $f_{\alpha} \subset L^{\beta}$ it is sufficient to show the existence of a number $A$ such that

$$
\left|\int_{0}^{2 \pi} \sigma_{n}\left[f_{\alpha}\right] g(-x) d x\right| \leqq A
$$

for every (periodic) $g$ with $\mathfrak{M}_{\beta^{\prime}}(g) \leqq 1$. It is well known that

$$
\begin{aligned}
& f(x) \sim \sum_{n=-\infty}^{\infty} c_{n} e^{i n x} \quad\left(c_{0}=0\right) \quad \text { implies } \\
& f_{\alpha}(x) \sim \sum_{n=-\infty}^{\infty} c_{n}|n|^{-\alpha} \exp \left(-\frac{\pi i \alpha}{2} \operatorname{sg} n\right) e^{i n x} .
\end{aligned}
$$

From this it is easily seen that the left-hand member of (8) is equal to

t It may be added that Theorems 1 and 2 are valid in the case of the Riemann-Liouville definition, at least if we suppose that the interval of integration is finite. The use of arithmetical means in the proof below of Theorem 2 is not essential and could easily be avoided. 


$$
\left|\int_{0}^{2 \pi} f(-x) \sigma_{n}\left[g_{\alpha}\right] d x\right| .
$$

Using W. H. Young's inequality $†$ we see that this expression does not exceed

$$
\int_{0}^{2 \pi} \Phi(|f|) d x+\int_{0}^{2 \pi} \Psi\left(\left|\sigma_{n}[g]\right|\right) d x
$$

where $\Phi$ and $\Psi$ are conjugate. We take

$$
\Psi(x)=\exp \left(\lambda x^{\beta}\right)-1,
$$

$\lambda$ being the same constant as occurs in Theorem 1. Since $\Psi$ is convex, we have by the inequality of Jensen and the inequality (4)

$$
\begin{aligned}
\Psi\left(\left|\sigma_{n}\left[g_{\alpha}(x)\right]\right|\right) & \leqq \Psi\left(\frac{1}{\pi} \int_{0}^{2 \pi}\left|g_{\alpha}(x+t)\right| K_{n}(t) d t\right) \\
& \leqq \frac{1}{\pi} \int_{0}^{2 \pi} \Psi\left(\left|g_{\alpha}(x+t)\right|\right) K_{n}(t) d t \\
\int_{0}^{2 \pi} \Psi\left(\left|\sigma_{n}\left(g_{\alpha}\right)\right|\right) d x & \leqq \int_{0}^{2 \pi} \Psi\left(\left|g_{\alpha}\right|\right) d x<\int_{0}^{2 \pi} \exp \lambda\left|g_{\alpha}\right|^{\beta} d x<\Lambda,
\end{aligned}
$$

where $K_{n}(t)$ denotes the Fejér kernel. It follows from (11) that, for $y$ large, the conjugate function $\Phi(y)$ is asymptotically equal to $\lambda^{-1 / \beta} y(\log y)^{1 / \beta}$, and so the first term in (10) has a finite value M. Consequently (8) is true with $A=\Lambda+\mathrm{M}$ and Theorem 2 is established.

4. We now prove

Theorem 3. If $0<\alpha \leqq 1$ and $f \subset L^{1, \alpha}$, the (complex) Fourier coefficients $c_{n}$ of $f$ satisfy the inequality

$$
\left(\sum_{n=1}^{\infty} n^{-1}\left|c_{n}\right|^{1 / \alpha}\right)^{\alpha}<A_{\alpha} \int_{0}^{2 \pi}|f|\left(\log ^{+}|f|\right)^{\alpha} d x+B_{\alpha},
$$

with $A_{\alpha}$ and $B_{\alpha}$ depending only on $\alpha$. For $\alpha>1$ the theorem is false.

$\dagger$ Let $\phi(x), x \geqq 0$, be a continuous increasing function, with $\phi(0)=0$, and let $\psi(y)$ be the function inverse to $\phi(x)$. If

$$
\Phi(x)=\int_{0}^{x} \phi(u) d u, \Psi(y)=\int_{0}^{y} \psi(v) d v,
$$

then, for every $a \geqq 0, b \geqq 0$, we have

$$
a b \leqq \Phi(a)+\Psi(b) .
$$

The sign $\leqq$ in $\left(^{*}\right)$ degenerates into $=$ if, and only if, $b=\phi(a)$. The functions $\Phi$ and $\Psi$ are called conjugate, of course, in the sense different from that used in the theory of Fourier series. For a very simple proof of Young's inequality $\left(^{*}\right)$ see Oppenheim [16]. 
We assume for simplicity that $f$ is real and so $c_{-n}=\bar{c}_{n}$. Similarly, although we suppose in the proof that $c_{0}=0$, the inequality (13) remains valid without this assumption. From (9) and Theorem 2 it follows that

$$
f_{1-\alpha}(x) \sim \sum_{n=-\infty}^{\infty} c_{n}|n|^{\alpha-1} \epsilon_{n} e^{i n x} \subset L^{1 / \alpha}, \quad\left|\epsilon_{n}\right|=1 .
$$

Now we use the following theorem of Hardy and Littlewood [6]:

If $\phi \sim \sum \gamma_{n} e^{i n x} \subset L^{p}, 1<p \leqq 2$, then

$$
\sum_{n=1}^{\infty}\left|\gamma_{n}\right|^{p} n^{p-2}<A_{p} \int_{0}^{2 \pi}|\phi|^{p} d x
$$

with $A_{p}$ independent of $\phi$.

Applying this theorem, with $p=1 / \alpha, \phi=f_{1-\alpha}$, to the series (14) and taking into account (7), we obtain (13) for $\frac{1}{2} \leqq \alpha<1$. We shall not consider here the case $\alpha=1 \dagger$, and, for $0<\alpha<\frac{1}{2}$, since the inequality (13) can be strengthened $\dagger$, we shall be contented with proving the convergence of the series (13).

Lemma. Let $f(x), g(x)$ be non-negative in $(0,2 \pi)$ and let $\phi(u), \psi(u), u \geqq 0$, be two non-negative and non-decreasing convex functions. Put

$$
\chi(u)=\phi(\psi(u)), \quad h(x)=\int_{0}^{2 \pi} f(t) g(x+t) d t .
$$

If

then

$$
\int_{0}^{2 \pi} f(x) d x=1, \quad \int_{0}^{2 \pi} \psi(g(x)) d x \leqq 1
$$

$$
\int_{0}^{2 \pi} \chi(h(x)) d x \leqq \int_{0}^{2 \pi} \phi(f(x)) d x .
$$

Let $1 / \kappa, \kappa \geqq 1$, be the value of the second integral in (17). Using twice Jensen's inequality, we have

$$
\begin{aligned}
\chi(h(x)) & =\phi\left[\psi\left(\int_{0}^{2 \pi} f(t) g(x+t) d t\right)\right] \leqq \phi\left[\int_{0}^{2 \pi} f(t) \psi(g(x+t)) d t\right] \\
& \leqq \phi\left[\int_{0}^{2 \pi} \kappa f(t) \psi(g(x+t)) d t\right] \leqq \int_{0}^{2 \pi} \phi(f(t)) \kappa \psi(g(x+t)) d t, \\
\int_{0}^{2 \pi} \chi(h(x)) d x & \leqq \int_{0}^{2 \pi} \phi(f(t)) d t \int_{0}^{2 \pi} \kappa \psi(g(t)) d t=\int_{0}^{2 \pi} \phi(f(t)) d t .
\end{aligned}
$$

† See the next Note VI. 
Corollary. If $f \subset L^{1, \alpha}, g \subset L^{1, \beta}, \alpha \geqq 0, \beta \geqq 0$, then $h \subset L^{1, \alpha+\beta}$.

Let $\phi(u)=u\left(\log ^{+} u\right)^{\alpha}, \psi(u)=u\left(\log ^{+} u\right)^{\beta}$. We may plainly assume that conditions (17) are satisfied. Then it is sufficient to notice that, for $u \geqq u_{0}$, we have

$$
\chi(u)=u(\log u)^{\beta}\{\log (u \log \beta u)\}^{\alpha} \geqq u(\log u)^{\alpha+\beta} .
$$

Suppose now that $\frac{1}{1} \leqq \alpha<\frac{1}{2}$ and set $g=f$ in the integral (16). It is well known that then

$$
h(x) \sim 2 \pi \sum_{n=1}^{\infty}\left|c_{n}\right|^{2} e^{i n x} .
$$

Since $h \subset L^{1,2 \alpha}$ and $\frac{1}{2} \leqq 2 \alpha<1$, we obtain, by applying Theorem 3 in the case already established, the convergence of the series

$$
\sum_{n=1}^{\infty} n^{-1}\left|c_{n}\right|^{2 \cdot 1 /(2 \alpha)}=\sum_{n=1}^{\infty} n^{-1}\left|c_{n}\right|^{1 / \alpha}
$$

We proceed similarly when $\frac{1}{8} \leqq \alpha<\frac{1}{4}$, and so on.

In order to show that the condition $0<\alpha \leqq 1$ cannot be removed, consider the function

$$
f(x)=\sum_{n=2}^{\infty}(\log n)^{-\alpha}\left(\log _{2} n\right)^{-\beta} \cos n x .
$$

It may be shown that in the neighborhood of $x=0^{+}$,

$$
f(x)=O\left[x^{-1}(\log 1 / x)^{-(\alpha+1)}\left(\log _{2} 1 / x\right)^{-\beta}\right],
$$

and, consequently, $f \subset L^{1, \alpha}$ if only $\beta>1$. If, moreover, $1<\beta<\alpha$, the series (18) diverges. To get the needed estimate observe that, by Abel's transformation,

$$
f(x) \equiv \sum_{n=2}^{\infty} a_{n} \cos n x=\sum_{n=2}^{\infty} \Delta^{2} a_{n} \frac{\sin ^{2}(n+1) \frac{x}{2}}{4 \sin ^{2} \frac{x}{2}} .
$$

Now we break up the last sum into two, the first being extended over the range $2 \leqq n \leqq 1 / x$. In the first sum the coefficient of $\Delta^{2} a_{n}$ is $O\left(n^{2}\right)$, in the second it is $O\left(x^{-2}\right)$. It simplifies slightly the proof if we use the fact that $\Delta^{2} a_{n} \geqq 0$ for $n \geqq n_{0}$. 


\section{SOME THEOREMS ON FOURIER COEFFICIENTS}

1. Given a sequence of (complex) numbers $c_{1}, c_{2}, \cdots, c_{n}, \cdots$, we shall denote by $c_{1}{ }^{*}, c_{2}{ }^{*}, \cdots, c_{n}{ }^{*}, \cdots$ the sequence $\left|c_{1}\right|,\left|c_{2}\right|, \cdots,\left|c_{n}\right|, \cdots$ rearranged in descending order of magnitude.

Hardy and Littlewood [9] have established the following theorems. $\dagger$

Theorem A. Suppose that

$$
f(x) \sim \sum_{n=-\infty}^{\infty} c_{n} e^{i n x}, \quad c_{-n}=\bar{c}_{n}
$$

and that $|f| \log ^{+}|f| \subset L$. Then $\sum_{1}^{\infty} n^{-1} c_{n}^{*}$ is convergent and $\sum \exp \left(-k /\left|c_{n}\right|\right)$ is convergent for every $k>0$.

Theorem B. Suppose that $\sum_{1}^{\infty}\left|c_{n}\right|\left(\log \left(1 /\left|c_{n}\right|\right)\right)^{-1}$ is convergent. Then $\sum c_{n} e^{i n x}$ is the Fourier series of a function $f$, such that $\exp (k|f|)$ is integrable for every $k>0$.

Our object here is to generalize these theorems in two directions. First, we consider slightly more general types of integrability and, secondly, the results are extended to general, uniformly bounded, orthogonal systems.

Let $\phi_{2}, \phi_{3}, \ldots \ddagger$ be a system of functions, orthogonal and normal in a finite interval $(a, b)$ and uniformly bounded,

$$
\left|\phi_{n}(x)\right| \leqq M \text {. }
$$

These conditions will be assumed in the following discussion.

THEOREM 1. If $|f|\left(\log ^{+} f\right)^{\alpha} \subset L$ in $(a, b), \alpha>0$, then

(i) the series $\sum \exp \left(-k /\left|c_{n}\right|^{1 / \alpha}\right)$ converges for every $k>0$.

(ii) If, moreover, $\alpha \leqq 1$, we have $\sum n^{-1} c_{n}{ }^{* 1 / \alpha}<\infty$.

Theorem 2. If the series $\sum\left|c_{n}\right|\left(\log \left(1 /\left|c_{n}\right|\right)\right)^{-\alpha}, \alpha>0$, converges, the series

$$
\sum_{n=2}^{\infty} c_{n} \phi_{n}(x)
$$

is the Fourier series of a function $f$ such that $\exp \left(k|f|^{1 / \alpha}\right)$ is integrable for every $k>0$.

$\dagger$ The results are stated without proofs. A result less strong than Theorem A, viz., the convergence of the series $\Sigma n^{-1}\left|c_{n}\right|$, is proved in Zygmund [29, Theorem 3]. Since the argument used there can be applied, with slight modifications, to general uniformly bounded orthogonal systems, it yields also the result of Hardy and Littlewood. The latter result is, in turn, contained in the following theorem: If $f(x) \sim c_{0}+c_{1} e^{i x}+\cdots+c_{n} e^{i n x}+\cdots$, then the series $\Sigma n^{-1} c_{n}{ }^{*}$ converges.

$\ddagger$ It is slightly more convenient to denote the system by $\phi_{2}, \phi_{3}, \cdots$, and not by $\phi_{1}, \phi_{2}, \cdots$. Correspondingly, $c_{2}{ }^{*}, c_{8}^{*}, \cdots$ denotes the sequence $\left|c_{2}\right|,\left|c_{3}\right|, \cdots$ rearranged in descending order. 
2. The proof will be based on a series of lemmas.

LEMMA 1. Let $y=\phi(x), x \geqq 0$, be a non-negative, continuous, strictly increasing function with $\phi(0)=0$. Let $x=\psi(y)$ be the function inverse to $\phi(x)$. Then, for every $a, b \geqq 0$, the inequality

(3) $a b \leqq \Phi(a)+\Psi(b), \quad \Phi(x)=\int_{0}^{x} \phi(u) d u, \quad \Psi(y)=\int_{0}^{y} \psi(v) d v$

holds. The sign of equality occurs in (3) if, and only if, $b=\phi(a)$. (Cf. footnote $\dagger$ on page 607.)

LEMMA 2. Let

where

$$
f(x) \sim \sum_{n=2}^{\infty} c_{n} \phi_{n}(x)
$$

$$
\left|c_{n}\right| \leqq n^{-1}(\log n)^{\alpha-1}, \quad \alpha>0, \quad n=2,3, \cdots .
$$

Then, for $\lambda>0$ sufficiently small, we have

$$
\int_{a}^{b} \exp \left(\lambda|f|^{1 / \alpha}\right) d x \leqq A . \dagger
$$

The function $(\log x)^{\alpha-1}$ decreases for $x>e^{\alpha-1}$. Let $n_{0}$ be an integer $>e^{\alpha-1}$. Without loss of generality we may assume that $c_{n}=0$ for $n \leqq n_{0}$. If $\mu \geqq 2$, we have, by the F. Riesz theorem (cf. M. Riesz [21]),

$$
\begin{aligned}
\int_{a}^{b}|f|^{\mu} d x & \leqq M^{\mu-2}\left[\sum_{n=n_{0}+1}^{\infty} n^{-\mu^{\prime}}(\log n)^{(\alpha-1) \mu^{\prime}}\right]^{\mu-1} \\
& \leqq M^{\mu-2}\left(\int_{2}^{\infty} x^{-\mu^{\prime}}(\log x)^{(\alpha-1) \mu^{\prime}} d x\right)^{\mu-1}
\end{aligned}
$$

The last factor does not exceed $A^{\mu-1}$. where $A=A_{\alpha}$ is a constant independent of $\mu$, if only $\mu \geqq \mu_{0} \geqq 2$.

Put $\mu=\beta k$, where $\beta=1 / \alpha$. Let $k_{0}$ denote an integer, such that $\beta k \geqq \mu_{0}$ for $k \geqq k_{0}$. Then

$$
\frac{\lambda^{k}}{k !} \int_{a}^{b}|f|^{\beta k} d x \leqq M^{-2} A^{-1} \lambda^{k}(A M)^{\beta k}(\beta k)^{k} / k !, \quad k \geqq k_{0},
$$

and, by Stirling's formula, we obtain

† We designate by $A$ any constant (not necessarily the same in all the formulas) which does not depend on $f$. 


$$
\int_{a}^{b} \sum_{k=k_{0}}^{\infty}(k !)^{-1}\left(\lambda|f|^{\beta}\right)^{k}<A
$$

if only $\lambda e A^{\beta} M^{\beta} \beta<1$. Since

$$
\sum_{k=0}^{k_{0}-1}(k !)^{-1}(\lambda u)^{\beta k}<\sum_{k=k_{0}}^{\infty}(k !)^{-1}(\lambda u)^{\beta k}
$$

for $u \geqq u_{0}$, the inequality (4) follows from (5).

LEMMA 3. If $|f|\left(\log ^{+}|f|\right)^{\alpha} \subset L, \alpha>0$, and if $c_{n}, n=2,3, \cdots$, are the Fouriex coefficients of $f$ with respect to $\left\{\phi_{n}\right\}$, then

$$
\sum_{n=2}^{\infty} n^{-1}(\log n)^{\alpha-1} c_{n}^{*} \leqq A \int_{a}^{b}|f|\left(\log ^{+}|f|\right)^{\alpha} d x+A .
$$

Since the order of the functions $\phi_{n}$ is irrelevant, we may suppose that $c_{n}{ }^{*}=\left|c_{n}\right|$. Put $\epsilon_{n}=\overline{\operatorname{sg} c_{n}}$ and consider the partial sums $s_{N}$ of the series

$$
\sum_{n=2}^{\infty} \epsilon_{n} n^{-1}(\log n)^{\alpha-1} \phi_{n}(x) .
$$

Using Young's inequality we obtain

(8) $\sum_{n=2}^{N} n^{-1}(\log n)^{\alpha-1} c_{n}^{*}=\int_{a}^{b} f(x) s_{N}(x) d x \leqq \int_{a}^{b} \Phi(|f|) d x+\int_{a}^{b} \Psi\left(\left|s_{N}\right|\right) d x$.

Put

$$
\begin{aligned}
& \Psi(x)=x \exp \left(\lambda_{0} x^{\beta}\right)-x, \quad \beta=1 / \alpha, \text { and hence } \\
& \Phi(x) \sim\left(\lambda_{0}\right)^{-\alpha} x(\log x)^{\alpha} \text { as } x \rightarrow \infty,
\end{aligned}
$$

where $\lambda_{0}$ is any positive constant less than the constant $\lambda$ occurring in (4). Since $\Psi(x) \leqq \exp \left(\lambda x^{\beta}\right), x \geqq x_{0}$, we get, from (8) and (4),

$$
\sum_{n=2}^{\infty} n^{-1}(\log n)^{\alpha-1} c_{n}^{*} \leqq \int_{a}^{b} \Phi(|f|) d x+A .
$$

Since $\Phi(x)<2 \lambda_{0}{ }^{-\alpha} x(\log x)^{\alpha}, x \geqq x_{0}$, (6) follows from (10).

3. Now it is not difficult to prove Theorem 1 . Let $B_{0}$ denote the righthand side of (6). Then

$$
c_{n}^{*} \sum_{\nu=2}^{n} \nu^{-1}(\log \nu)^{\alpha-1} \leqq \sum_{\nu=2}^{n} c_{\nu}^{*} \nu^{-1}(\log \nu)^{\alpha-1}<B_{0} .
$$

Since the coefficient of ${c_{n}}^{*}$ in the first term is $\geqq \rho(\log n)^{\alpha}, \rho$ being a constant 
independent of $n$, the following three inequalities are consequences of (11):

$$
c_{n}^{*} \leqq B_{0} \rho^{-1}(\log n)^{-\alpha}, \quad \log n \leqq\left(B_{0} /\left(\rho c_{n}^{*}\right)\right)^{\beta}, \quad n \leqq \exp \left(B_{0} /\left(\rho c_{n}^{*}\right)\right)^{\beta} .
$$

From the second of them and from (6) we get

$$
\left(\sum_{n=2}^{\infty} n^{-1} c_{n}^{* 1 / \alpha}\right)^{\alpha} \leqq \rho^{\alpha-1} B_{0}=\rho^{\alpha-1}\left\{A \int_{a}^{b}|f|\left(\log ^{+}|f|\right)^{\alpha} d x+A\right\},
$$

which is the second part of Theorem $1 . \dagger$ To prove the first part, we notice that the function $x^{-1}(\log x)^{\alpha-1}$ decreases for $x \geqq n_{0} \geqq e^{\alpha-1}$, and so, from the third inequality (12) and (6), we obtain

$$
\begin{gathered}
n^{-1}(\log n)^{\alpha-1} \geqq\left\{B_{0} /\left(\rho c_{n}^{*}\right)\right\}^{\beta(\alpha-1)} \exp \left(-B_{1} / c_{n}^{* \beta}\right), \quad B_{1}=\left(B_{0} / \rho\right)^{\beta}, \quad n \geqq n_{0}, \\
\sum_{n=n_{0}}^{\infty}\left(c_{n}^{*}\right)^{2-1 / \alpha} \exp \left(-B_{1}\left(c_{n}^{*}\right)^{-1 / \alpha}\right) \leqq \rho^{(\alpha-1) / \alpha} B_{0}^{1 / \alpha} .
\end{gathered}
$$

This gives statement (i) of Theorem 1 , for some $k>0$. To prove it for every $k>0$ it suffices to notice (rejecting a large number of terms from the convergent series (10)) that $c_{n}=o\left((\log n)^{-\alpha}\right)$, and to repeat the previous argument.

4. We now pass to Theorem 2 .

LEMMA 4. Let $c_{n}>0, b_{n} \geqq b_{n+1}>0, \alpha>0$, and

$$
\sum_{n=3}^{\infty} c_{n}\left(\log \frac{1}{c_{n}}\right)^{-\alpha} \leqq C_{\alpha}<\infty, \quad \sum_{n=3}^{\infty} n^{-1}(\log n)^{\alpha-1} b_{n} \leqq B_{\alpha}<\infty .
$$

There exists a number $\sigma>0$ depending only on $\alpha$, and such that

$$
S=\sum_{n=3}^{\infty} c_{n} b_{n} \leqq\left(\sigma C_{\alpha}+\frac{1}{2}\right) B_{\alpha} .
$$

From the second inequality it follows that $b_{n} \leqq B_{\alpha} \rho^{-1}(\log n)^{-\alpha}$. Break up the sum $S$ into two, $S=S_{1}+S_{2}$, where $S_{1}$ contains the indices $n$ for which $b_{n} \leqq \sigma B_{\alpha}\left(\log \left(1 / c_{n}\right)\right)^{-\alpha}, \sigma$ being defined by the equality $(\rho \sigma)^{\beta}=3$. It is obvious that $S_{1} \leqq \sigma C_{\alpha} B_{\alpha}$. If $n$ occurs in $S_{2}$, we have

$$
B_{\alpha} \rho^{-1}(\log n)^{-\alpha} \geqq b_{n} \geqq B_{\alpha} \sigma\left(\log 1 / c_{n}\right)^{-\alpha},
$$

and hence $c_{n} \leqq n^{-3}$. Therefore

$$
\begin{aligned}
S_{2} \leqq \sum_{n=3}^{\infty} n^{-3} b_{n} & \leqq \sum_{n=3}^{\infty} n^{-2}(\log n)^{-1} b_{n} \leqq \frac{1}{2} \sum_{n=3}^{\infty} n^{-1}(\log n)^{-1} b_{n} \\
& \leqq \frac{1}{2} \sum_{n=3}^{\infty} n^{-1}(\log n)^{\alpha-1} b_{n}=\frac{B_{\alpha}}{2}
\end{aligned}
$$

$\dagger$ The condition $\alpha \leqq 1$ is essential. See $V$, Theorem 3 . 
and the lemma follows.

Suppose now that in the series (2) we have not only $c_{3}=0$, but that also a number of subsequent coefficients vanish, $c_{4}=\cdots=c_{n_{0}}=0, n_{0}$ being so large that

$$
C_{0}=\sum_{n=n_{0}+1}^{\infty}\left|c_{n}\right|\left(\log 1 /\left|c_{n}\right|\right)^{-\alpha} \leqq 1 /(2 \sigma) .
$$

It follows that the coefficient of $B_{\alpha}$ in (14) does not exceed 1 if $C_{\alpha}$ is replaced there by $C_{0}$. Let $s_{N}, N>n_{0}$, denote the partial sums of the series (2), and let $g$ be any function with Fourier coefficients $b_{n}$ and $\Phi(|g|)$ integrable. Then

$$
\left|\int_{a}^{b} s_{N} g d x\right|=\left|\sum_{n=n_{0}+1}^{N} c_{n} b_{n}\right| \leqq \sum_{n=n_{0}+1}^{\infty}\left|c_{n}\right|\left|b_{n}\right| \text {. }
$$

On rearranging the terms in the last sum according to the decreasing magnitude of $\left|b_{n}\right|$ and applying Lemma 4, and the inequality (10) (with a slightly different notation where $c_{n}{ }^{*}, f$ have been replaced by $\left.b_{n}{ }^{*}, g\right)$, we get

$$
\left|\int_{a}^{b} s_{N} g d x\right| \leqq \sum_{n=2}^{\infty} n^{-1}(\log n)^{\alpha-1} b_{n}^{*} \leqq \int_{a}^{b} \Phi(|g|) d x+D .
$$

On the other hand, if $g$ is chosen conveniently (see Lemma 1) the left-hand member in (15) is equal to

$$
\int_{a}^{b} \Psi\left(\left|s_{N}\right|\right) d x+\int_{a}^{b} \Phi(|g|) d x
$$

the last integral being finite. Comparing this with the right-hand side of (15), we get

$$
\int_{a}^{b}\left|\Psi\left(\left|s_{N}\right|\right)\right| d x \leqq D .
$$

By the theorem of Riesz-Fischer, the series (2) is the Fourier series of a function $f \subset L^{2}$ and a subsequence of $\left\{s_{N}\right\}$ converges almost everywhere to $f$. By Fatou's well known lemma, the inequality (16) implies

$$
\int_{a}^{b} \Psi(|f|) d x \leqq D .
$$

It follows that $\exp \left(k|f|^{\beta}\right)$ is integrable for some $k>0$. Rejecting the restriction concerning the first coefficients of the series, we may assert the integrability of $\exp \left(k\left|f-s_{n_{0}}\right|^{\beta}\right)$, where $n_{0}$ is sufficiently large. Since $s_{n_{0}}$ is bounded, $\exp \left(k|f|^{\beta}\right)$.is again integrable for some $k>0$. 
To prove that it is integrable for every $k>0$, it suffices to observe that for any $\lambda>0$ and $c_{n}^{\prime}=\lambda c_{n}$ the series $\sum\left|c_{n}^{\prime}\right|\left(\log \left(1 /\left|c_{n}^{\prime}\right|\right)\right)^{-\alpha}$ converges and so $\exp \left(k \lambda|f|^{\beta}\right)$ is integrable for every $\lambda>0$.

5. We now prove

Theorem 3. If $\sum n^{r-1}\left|c_{n}\right|^{r}, r>1$, converges, the series (2) is the Fourier series of a function $f$ such that $\exp \left(k|f| r^{\prime}\right)$ is integrable for all values of $k>0$.

We shall only sketch the proof, which is analogous to, and even a little simpler than, that of Theorem 2. Using Hölder's inequality we see that the series $\sum c_{n} b_{n}$ converges, even absolutely, for any $\left\{b_{n}\right\}$, such that $\sum n^{-1}\left|b_{n}\right| r^{\prime}<\infty$. In particular, it converges if $b_{n}$ are the Fourier coefficients of a function $g$ such that $|g|\left(\log ^{+}|g|\right)^{1 / r^{\prime}}$ is integrable (see (iii) of Theorem 1). Since, roughly speaking, $\exp x^{r^{\prime}}$ and $x(\log x)^{1 / r^{\prime}}$ are conjugate in the sense of Young, the integrability of $\exp \left(k|f|^{r^{\prime}}\right)$ for some $k>0$, and hence for every $k>0$, follows.

Remark. In the case of trigonometric series and $r \geqq 2$, Theorem 3 is a corollary of Theorem 1 , Note V (using the inequality (15) of that note).

\section{On a theorem of Paley and Wiener}

In a recent paper Paley and Wiener [17] proved the following theorem: If $f(x)$ is defined over $(-\pi, \pi)$ as an odd function and is non-decreasing and integrable over $(-\pi, \pi)$, then its conjugate function $\tilde{f}(x)$ is also integrable. Here we propose to give a simpler proof of this theorem, or rather of an equivalent

Theorem. If $f(x)$ is odd in $(-\pi, \pi)$, non-increasing and integrable over $(0, \pi)$, then its conjugate function $\tilde{f}(x)$ is also integrable.

The theorem is trivial if $f(x)$ is bounded on $(0, \pi)$. On the other hand there is no loss of generality if we assume that $f(x)$ is not bounded only in the neighborhood of $x=0$ and that $f(x) \geqq 0,0<x \leqq \pi$. Our proof is based on the following obvious

LEMMA. If $f(x)$ is integrable over $(0, \pi)$ then the functions

$$
\begin{aligned}
& \phi(x)=x^{-2} \int_{0}^{x} t|f(t)| d t \\
& \psi(x)=\int_{x}^{\pi} t^{-1}|f(t)| d t
\end{aligned}
$$

are also integrable.

Now, assuming $0<x<\pi$, we have 


$$
\begin{aligned}
-\pi \tilde{f}(x) & =\int_{-\pi}^{\pi} f(t) \cot \frac{t-x}{2} d t \\
& =\int_{-x / 2}^{x / 2} \cdots+\int_{-3 x / 2}^{-x / 2} \cdots+\int_{x / 2}^{3 x / 2} \cdots+\left(\int_{-\pi}^{-3 x / 2} \cdots+\int_{3 x / 2}^{\pi} \cdots\right) \\
& \equiv J_{1}(x)+J_{2}(x)+J_{3}(x)+J_{4}(x) .
\end{aligned}
$$

Here

$$
\begin{aligned}
\left|J_{1}(x)\right| & \leqq \int_{0}^{x / 2} f(t) O\left(t x^{-2}\right) d t=O(\phi(x)) \subset L \\
\left|J_{2}(x)\right| & \leqq f\left(\frac{x}{2}\right) \int_{x / 2}^{3 x / 2} O\left(\frac{1}{t}\right) d t=O\left(f\left(\frac{x}{2}\right)\right) \subset L \\
\left|J_{4}(x)\right| & =O\left(\int_{3 x / 2}^{\pi} \frac{f(t) d t}{t}\right)=O\left(\psi\left(\frac{3 x}{2}\right)\right) \subset L, \\
\int_{0}^{\pi / 2}\left|J_{3}(x)\right| d x & =O\left\{\int_{0}^{\pi / 2} d x \int_{0}^{x / 2}|f(x+t)-f(x-t)| \frac{d t}{t}\right\} \\
& =O\left\{\int_{0}^{\pi / 4} \frac{d t}{t} \int_{2 t}^{\pi / 2}|f(x+t)-f(x-t)| d x\right\} \\
& =O\left\{\int_{0}^{\pi / 4} \frac{d t}{t}\left[\int_{t}^{\pi / 2-t} f(x) d x-\int_{3 t}^{\pi / 2+t} f(x) d x\right]\right\} \\
& =O\left\{\int_{0}^{\pi / 4} \frac{d t}{t}\left[\int_{t}^{3 t} f(x) d x-\int_{\pi / 2-t}^{\pi / 2+t} f(x) d x\right]\right\} \\
& =O\left\{\int_{0}^{\pi / 4} \frac{d t}{t}[2 t f(t)+O(t)]\right\}<\infty .
\end{aligned}
$$

This proves that $f(x)$ is integrable over $(0, \pi)$.

\section{BIBLIOGRAPHY}

1. T. J. Boks, Sur les rapports entre les méthodes d'integration de Riemann et de Lebesgue, Rendiconti del Circolo Matematico di Palermo, vol. 45 (1921), pp. 211-264.

2. A. Denjoy, Sur l'integration riemannienne, Comptes Rendus, vol. 169 (1919), pp. 219-221.

3. L. Fejér, Ueber die arithmetischen Mittel erster Ordnung der Fourierreihe, Göttinger Nachrichten, 1925, pp. 13-17.

4. L. Fejér and F. Riesz, Ueber einige funktionentheoretische Ungleichungen, Mathematische Zeitschrift, vol. 11 (1921), pp. 305-314.

5. G.H. Hardy, Remarks on three recent notes in the Journal, Journal of the London Mathematical Society, vol. 3 (1928), pp. 166-169.

6. G. H. Hardy and J. E. Littlewood, Some new properties of Fourier constants, Mathematische Annalen, vol. 97 (1927), pp. 159-209. 
7. G. H. Hardy and J. E. Littlewood, Some properties of fractional integrals, Mathematische Zeitschrift, vol. 27 (1927-1928), pp. 565-606.

8. G. H. Hardy and J. E. Littlewood, On absolute convergence of Fourier series, Journal of the London Mathematical Society, vol. 3 (1928), pp. 250-253.

9. G. H. Hardy and J. E. Littlewood, Some new properties of Fourier constants, Journal of the London Mathematical Society, vol. 6 (1931), pp. 3-9.

10. E. Hille, Note on the behavior of certain power series on the circle of convergence, Proceedings of the National Academy of Sciences, vol. 14 (1928), pp. 217-220.

11. E. Hille, Note on a power series considered by Hardy and Littlewood, Journal of the London Mathematical Society, vol. 4 (1929), pp. 176-182.

12. A. Kolmogoroff, Une série de Fourier-Lebesgue divergente presque partout, Fundamenta Mathematicae, vol. 4 (1923), pp. 324-328.

13. A. Kolmogoroff, Sur les fonctions harmoniques conjuguées et sur les séries de Fourier, Fundamenta Mathematicae, vol. 7 (1925), pp. 23-28.

14. A. Kolmogoroff, Sur un procédé d'intégration de M. Denjoy, Fundamenta Mathematicae, vol. 11 (1928), pp. 27-28.

15. Marcinkiewicz, Sur la divergence des séries de Fourier, to appear in the Fundamenta Mathematicae.

16. A. Oppenheim, Note on Mr. Cooper's generalization of Young's inequality, Journal of the London Mathematical Society, vol. 2 (1927), pp. 21-23.

17. R. E. A. C. Paley and N. Wiener, Notes on the theory and application of Fourier transforms, Note II, these Transactions, vol. 35 (1933), pp. 354-355.

18. R.E. A. C. Paley and A.Zygmund, On the partial sums of Fourier series, Studia Mathematica, vol. 2 (1930), pp. 221-227.

19. B. N. Prasad, On the summability of Fourier series and the bounded variation of power series, Proceedings of the London Mathematical Society, (2), vol. 36 (1933), pp. 407-424.

20. F. Riesz, Sur les polynômes trigonométriques, Comptes Rendus, vol. 158 (1914), pp. 16571661.

21. M. Riesz, Sur les maxima des formes bilinéaires et sur les fonctionnelles linéaires, Acta Mathematica, vol. 49 (1926), pp. 465-497.

22. M. Riesz, Sur les fonctions conjugukes, Mathematische Zeitschrift, vol. 27 (1927-1928), pp. 218-244.

23. O. Szász, Ueber den Konvergenzexponenten der Fourierschen Reihen gewisser Funktionenklassen, Sitzungsberichte der Bayerischen Akademie der Wissenschaften, Mathematisch-Physikalische Klasse, 1922, pp. 135-150.

24. G. Szegö, Ueber einen Satz der Herrn Serge Bernstein, Schriften der Königsberger gelehrten Gesellschaft, Naturwissenschaftliche Klasse, vol. 5 (1928), pp. 59-70.

25. E. C. Titchmarsh, On conjugate functions, Proceedings of the London Mathematical Society, (2), vol. 29 (1929), pp. 49-80.

26. Z. Waraszkiewicz, Remarque sur un theorème de $M$. Zygmund, Bulletin International de l'Académie Polonaise, Classe des Sciences Mathématiques et Naturelles, (A), 1929, pp. 275-279.

27. H. Weyl, Bemerkungen zum Begriff der Differentialquotienten gebrochener Ordnung, Vierteljahrsschrift der Naturforschenden Gesellschaft in Zürich, vol. 62 (1917), pp. 296-302.

28. A. Zygmund, Remarque sur la convergence absolue des séries de Fourier, Journal of the London Mathematical Society, vol. 3 (1928), pp. 194-196.

29. A. Zygmund, Sur les fonctions conjuguees, Fundamenta Mathematicae, vol. 13 (1929), pp. 284-303.

30. A. Zygmund, On a theorem of Privaloff, Studia Mathematica, vol. 3 (1931), pp. 239-2477.

UNIVERSITY OF VILNA, Vilna, Poland 